delivery and consumption. It seems that SGA neonates manage to develop compensatory mechanisms probably with an increase of their brain perfusion.

\section{PO-0423 DIFFERENCES IN CEREBRAL OXYGENATION AND PERFUSION OF SGA NEONATES ACCORDING TO GESTATIONAL AGE DURING THE FIRST POSTNATAL WEEK}

E Milona, P Karagianni, C Tsakalidis, D Rallis, G Mitsiakos, N Nikolaidis. 2nd Neonatal Departement, Aristotle University of Thessaloniki, Thessaloniki, Greece

\subsection{6/archdischild-2014-307384.1066}

Background and aims Low birth weight in conjuction with the immature autoregulation mechanisms noted in preterm neonates may influence brain perfusion and consequently the neurodevelopmental outcome.

The aim of this study was to assess the potential variations in brain oxygenation and perfusion in SGA neonates of different gestational age during the first postnatal week with the use of Near Infrared Spectroscopy (NIRS).

Patients/methods SGA neonates born at 28-32 weeks and $32^{+1}$ 36 weeks respectively consisted the two study groups. Three measurements of forty minutes each were performed with NIRO-200 NX instrument in the first, third and seventh day of life. Tissue oxygenation index (TOI), fraction tissue oxygen extraction (FTOE) and tissue haemoglobin index (THI) were the recorded parameters.

Results 38 neonates were prospectively enrolled; 12 were born at 28-32 weeks gestation and 26 at $32^{+1}-36$ weeks. Mean birth weight and head circumference were $1.062 \pm 265$ vs $1.656 \pm$ 401 and $26.0 \pm 2.5$ vs $30.4 \pm 1.8$ respectively in the study populations. Haemoglobin in the first day didn't show any significant difference between the two subgroups (16.4 vs 17.8). NIRS monitoring found higher FTOE at the right side in more preterm SGA neonates $(p=0.018)$ in the first postnatal day. All the other parameters were similar in the two groups.

Conclusion It seems there aren't significant differences in cerebral oxygenation and perfusion between SGA neonates of different gestational age during the first postnatal week.

\section{PO-0424 DOES SUPPLEMENTARY OXYGEN DURING A DESATURATION CAUSE CEREBRAL HYPEROXIA IN PRETERM NEONATES?}

KCW Moerman, TE Schat, AGJFvan Zoonen, AF Bos, EMW Kooi. Neonatology, Beatrix Children's Hospital/University Medical Center Groningen, Groningen, Netherlands

\subsection{6/archdischild-2014-307384.1067}

Background Supplementary oxygen is often administered when preterm neonates experience desaturations i.e. after apnea, sometimes resulting in cerebral hyperoxia during recovery. Whether this post-hypoxic cerebral hyperoxia is induced by the supplementary oxygen, remains unknown.

Aim To compare the regional cerebral oxygen saturation $\left(\mathrm{rcSO}_{2}\right)$ and cerebral fractional oxygen extraction (cFTOE) following a desaturation, between preterm neonates who did and did not receive supplementary oxygen.

Methods As part of a larger prospective cohort study, near infrared spectroscopy (NIRS) was used to measure $\mathrm{rcSO}_{2}$ during days 2 to 5 after birth. We collected 50 consecutive desaturations $\left(\mathrm{SpO}_{2}<80 \%\right)$. CFTOE was calculated: $\left[\left(\mathrm{SpO}_{2}-\mathrm{rcSO}_{2}\right) / \mathrm{SpO}_{2}\right]$. We used a Wilcoxon signed rank test to compare mean $\mathrm{rcSO}_{2}$ and cFTOE ten minutes before to ten minutes after desaturation. The Mann-Whitney $U$ test was used to compare these values in neonates who did and did not receive supplementary oxygen.

Results We included 50 desaturations in 16 preterm neonates (median GA 28+4/7 (range 25+0/7-30+0/7) weeks; birth weight 1144 (800-1630) grams). Supplementary oxygen was administered in $60 \%$ of the events. We found higher $\mathrm{rcSO}_{2}$ values following desaturation (mean 76.9\%) compared to before desaturation (mean $70.2 \%, \mathrm{p}=0.001$ ). There was no difference in $\mathrm{rcSO} 2$-increase between neonates who did and who did not receive supplementary oxygen $(\mathrm{p}=0.79)$. CFTOE was lower after desaturation $(0.17 \%)$ compared to cFTOE before desaturation $(0.22 \%, \mathrm{p}<0.001)$.

Conclusion Cerebral hyperoxia after desaturation in preterm neonates indeed occurs, but is not induced by supplementary oxygen administration. The decrease of cFTOE after desaturation supports the hypothesis that post-hypoxic reperfusion might cause cerebral hyperoxia after desaturation.

\section{P0-0425 MOTOR DEVELOPMENT AND QUALITY OF MOTOR PERFORMANCE IN RELATION TO COGNITIVE ABILITY IN VERY PRETERM CHILDREN}

C Montgomery, Y Fredriksson Kaul, L Hellström Westas, K Strand Brodd, K Persson. Perinatal Neonatal and Pediatric Cardiology Research, Department of Women's and Children's Health, Uppsala, Sweden

\subsection{6/archdischild-2014-307384.1068}

Background and aim Early motor and cognitive development are interdependent. Poor motor ability decreases possibility to explore and interact with the surrounding environment. Very preterm infants (VPT) and extremely preterm infants (EPT) frequently display atypical motor performance during their first year but little is known whether this predicts later cognitive ability. Is level of motor development (LMD) and quality of motor performance (QMP) at 10 months corrected age (CA), with regards to gestational age (GA) and gender, associated with cognitive ability at $2 \frac{1}{2}$ years CA.

Method Very preterm infants ( $\mathrm{n}=85,48$ boys, 37 girls) born 2004-2007, with a mean of 28.5 (22.3-31.9) gestational weeks $($ EPT $\mathrm{n}=30$, VPT $\mathrm{n}=55)$ and birth weight 1188 (520-2030 g). Structured Observation of Motor Performance in Infants (SOMP-I), which measures LMD and QMP separately, was performed at 10 months CA. Cognitive ability was assessed with Bayley Scales of Infant Development III (BSID III) at age 2 1/2.

Results Statistically significant associations were shown between LMD ( $\mathrm{r}=0.344 ; \mathrm{p}=0.001)$ and QMP $(\mathrm{r}=-256 ; \mathrm{p}=0.018)$, respectively, and BSID III cognitive scaled scores. When separated for gender and GA significant associations were shown for boys $(\mathrm{r}=0.392 ; \mathrm{p}=0.006)$ and EPT children $(\mathrm{r}=0.531 ; \mathrm{p}=$ 0.003 ) for LMD only.

Conclusion Motor development at 10 months is associated with cognitive development at $2 \frac{1}{2}$ years. Level of motor performance had a stronger correlation with cognition than QMP, and when analysed for subgroups, was present only in boys and children born extremely preterm.

\section{PO-0426 WITHDRAWN}

